\title{
New Pathophysiology Insight of Gut Microbiota's Influence on Systemic Lupus Erythematosus: A Brief Review
}

\author{
Dewi Purwita Agustini
}

\section{ABSTRACT}

Systemic lupus erythematosus (SLE) is a commonly found chronic autoimmune disease that affects multiple organs and systems. According to a new study, commensal gut microbiota promotes chronic autoimmunity in SLE patients with a lower Firmicutes/Bacteroidetes ratio. It has to do with the activation of inflammatory mediators such as toll-like receptors (TLRs), NOD-like receptors, adaptive immune response, and antimicrobial peptides mucins, defensins, and immunoglobulin A production. Aside from that, a decreased Firmicutes/Bacteroidetes ratio may cause $T$ helper cells and regulatory $T$ cells to become activated, both of which are linked to autoimmune activation in SLE. Theoretically, the immunomodulatory influence of food components in immune system activation has been the focus of nutrition for SLE patients, but probiotics and prebiotics have also been shown to dramatically enhance gut microbiota associated to SLE.

Published Online: January 24,2022

ISSN: 2684-5199

DOI : 10.24018 /ejbio.2022.3.1.321

\section{P. Agustini*}

Faculty of Medicine, Sanglah General Hospital, University of Udayana, Bali, Indonesia.

(e-mail: dewiagustinidr@gmail.com)

*Corresponding Author

Keywords: Autoimmune, chronic disease, gut microbiota, systemic lupus erythematosus.

\section{INTRODUCTION}

Systemic lupus erythematosus (SLE) is a commonly found chronic autoimmune disease characterized by diffuse connective tissue disorder that affects multiple organs and systems (Schulz \& Derk, 2009). SLE has a wide range of incidences across countries, with the global incidence in 2017 ranging above 50 to 241 per 100,000 adults (Rees et al., 2017). Aside from that, (Mu et al., 2017) reported that there were 20 to 200 cases per 100,000 patients among Asians, which affecting primarily women (but also quite common in males, children, and those over 50 years old), and targeting a variety of organs including the heart, kidney, skin, lung, and gastrointestinal tract (Mu et al., 2017; Han et al., 2015). This form of autoimmune disorder may recur and go into remission again, and typically holds of huge number of autoantibodies in the body. Apparently, the etiology of SLE is still unknown, but it has been linked to a variety of environmental (e.g., drug exposure and UV radiation), hormonal, and genetic variables.

Nonetheless, the present understanding of the relationship between gut microbiota and SLE has been demonstrated in a lupus-prone mouse model (Bandre et al., 2021). The gut microbiome has been recommended as another crucial component. However, an insufficiency of knowledge continues to cast doubt on proofing the impact of gut microbiota on SLE (Mahajan et al., 2021). There are various possibilities on how the gut microbiota interacts with the host and how is it related with SLE, but still no definitive theories have been found. Thus, the purpose of this review is to present the current ideas and thoughts of gut microbiota's role on SLE.

\section{Gut Microbiota AND AUtoimmunE}

In theory, the human gastrointestinal system comprises of more than 100 trillion microorganisms, the most of which are found in the colon. Dysbiosis (changes in gut microbial population) has been linked to several autoimmune and chronic inflammatory disorders, including rheumatoid arthritis, type 1 diabetes, inflammatory bowel disease, and, most recently, SLE (Hevia et al., 2014). Changes in the microbiota are influenced by a variety of factors, including gender, age, nutrition, race, stress, lifestyle (smoking habit, alcohol intake), medicine (antibiotics), and infections (Neuman \& Koren, 2017). According to research by Perry et al, a number of microbiota (such as Clostridium clusters XIVa \& IVa, Lactobacillus, Eubacterium, Bacteroidetes, Firmicutes, and Bifidobacterium) are assumed to be connected to gastrointestinal physiology (Perry et al., 2014). In the human body system, the gut microbiota plays a role in immune activation. When the gut microbiota is out of balance, it can stimulate innate immune responses including toll-like receptors (TLRs), NOD-like receptors, adaptive immunological responses, and the release of mucins, antimicrobial peptides, defensins, and immunoglobulin A (IgA), which can help the immune system mature (Hollister et al., 2014). The gut microbiota also had a role in the regulation and development of Peyer patches, germinal centers, and mesenteric lymph nodes (MLN), according to a research by (Kamada et al., 2014). Aside from that, the gut microbiota controls the growth of effector $\mathrm{T}$ cells and the production of cytokines that impact the T helper (Th17) cells and regulatory $\mathrm{T}$ (Treg) cells, all of which have a key role in autoimmune activation in SLE (Kamada et al., 2014). 


\section{GUT Microbiota IN SLE}

Systemic lupus erythematosus has been known as a systemic autoimmune disease characterized by the development of hyperactive and aberrant autoantibodies against nuclear and cytoplasmic antigens, as well as the activation of auto reactive inflammatory $\mathrm{T}$ cells and atypical proinflammatory cytokine release (Mu et al., 2015). Wei et al., (2019) stated that, the gut microbiota may play a critical role in SLE patients which can be seen by a much-reduced Firmicutes/Bacteroidetes ratio (Wei et al., 2019). Ma et al also proposed that the commensal gut microbiota may be a trigger for chronic autoimmunity in SLE patients (Ma et al., 2019). They also discovered that the feces of lupus mice can promote the development of anti-dsDNA antibodies and an inflammatory immunological response in recipient germ-free mice, which would upregulate the expression of SLE susceptibility genes (Ma et al., 2019).

Additionally, the relative abundance of Firmicutes is lower (Fig.1c) in SLE patients, while that of Bacteroidetes is higher, indicating a lower ratio of Firmicutes to Bacteroidetes than in healthy people (Hevia et al.,2014). The overgrowth of Ruminococcus gnavus (RG) in the gut microbiota of a few female SLE patients differing in disease activity was initially described by Azzouz et al. RG expansions in the gut reflected the severity of disease activity and were common in SLE patients (Azzouz et al., 2019).

According to (Mu et al., 2015), increased gut permeability (leaky gut) and gut microbial product translocation have been shown to favor lupus formation and disease progression. Increased intestinal permeability and L. reuteri translocation into inner organs were found in TLR7-transgenic spontaneous lupus mice (Fig. 1a) (Zegarra-Ruiz et al., 2019). As a result, Enterococcus gallinarum has been found in the mesenteric vein, gut-draining lymph nodes, livers, and spleens (Fig. 1b) (Manfredo et al., 2018). The ileal barrier was similarly compromised in E. gallinarum monocolonized mice, as demonstrated by downregulated mucus layer and antimicrobial defense markers (Manfredo et al., 2018). Both species have the ability to activate pDCs and secrete interferon (IFN- $\alpha$ ), which are major factors to SLE pathogenesis (Fig. 1a, d). Even if the SLE patient does not have any gastrointestinal symptoms, an impaired gut barrier is found in SLE patients (Thim-Uam et al., 2020). The high quantity of serum lipopolysaccharide (LPS), a cell wall component of gram-negative bacteria, which may be eliminated by antibiotics (e.g. vancomycin) therapy, together with the relief of lupus-like manifestations in MRL/lpr mice, may imply enhanced gut permeability in SLE patients (Mu et al., 2017).

Moreover, the plasma LPS level in SLE patients is strongly linked with anti-dsDNA antibody titers in the blood (Ogunrinde et al., 2019). Other indicators linked with gut barrier insufficiency, such as fecal calprotectin levels, serum soluble CD14a, and serum 1-acid glycoprotein, have also been found to be elevated in SLE patients (Azzouz et al., 2019). (Lopez et al., 2016) discovered that SLE patients have decreased levels of the Firmicutes to Bacteroidetes ratio
(Synergistetes). Consequently, Synergistetes has the ability to lower blood levels of interleukin (IL-6) (a proinflammatory cytokine) and may induce B1 cells to generate natural protective IgM anti-phosphorylcholine under physiological settings. This can be accomplished in a number of ways [clearing of apoptotic cells and cellular debris, elimination of oxidized lipids, inhibition of mitogen-activated protein kinase (MAPK) activation, and other proinflammatory mediators] (Gronwall et al., 2012). Aside from that, patients with SLE had a lower abundance of Lactobacillaceae and a higher richness of Lachnospiraceae (Mu et al., 2015).

Lactobacillus spp. and Lactobacillus reuteri have recently been shown to improve kidney function in rats suffering from lupus nephritis. Treatment with Lactobacillus spp. repaired intestinal permeability (altered before the beginning of nephritis), lowered inflammatory cytokines (i.e. IL-6 and IL-18), and boosted anti-inflammatory cytokines (i.e. IL-10, Transforming growth factor (TGF-)) and Tregs, according to the researchers. It also showed a reduction in $\operatorname{IgG} 2 \mathrm{a}$ (one of the primary immunological deposits) and IFN- levels, indicating that renal impairment was improving. However, no such evidence was seen in male mice, indicating that the microbiome's effect is sexually linked and dependent, and implying a role for sex hormones in the gut microbiota's regulatory function on lupus ( $\mathrm{Mu}$ et al., 2017; $\mathrm{Mu}$ et al., 2015).

\section{Potential tREATMENT FOR GUT MiCROBIOTA IN SLE}

\section{A. Diet as Strategy on Gut Microbiota}

The immunomodulatory influence of dietary components in immune system activation has been conceptually highlighted in the nutrition strategy for patients with SLE. Although little is known about the influence of diet on microbiota in SLE patients, there is some nutrition research that suggests that part of the dietary impact on SLE pathogenesis might be done by gut microbiota manipulation (Vieira et al., 2014). Regrettably, there isn't enough evidence to say if a dietary intervention can help prevent SLE recurrences. A few studies from other diseases with dysbiosis have shown the function of many dietary components in balancing particular gut microbiota quantities, which might be applied to SLE.

Ley et al., (2006) found a low-fat and low-carbohydrate diet can replace the gut microbiota balance by boosting the number of Bacteroidetes in SLE patients with high Firmicutes/Bacteroidetes ratios. Animal proteins and saturated fats in the diet have also been linked to a higher abundance of Bacteroidetes, according to (Yatsunenko et al., 2012). High consumption of whole grains, on the other hand, has been linked to a positive rise in Firmicutes/Bacteroidetes ratio in the gastrointestinal tract, and the administration of a low-fat/high-fiber diet has been linked to a positive increase in Firmicutes quantity (Martinez et al., 2013; Wu et al., 2011). De Filippo et al., (2010) discovered that long-term intake of diets rich in whole grains, dietary fibers, and vegetables resulted in decreased levels of Bacteroidetes in African children, demonstrating the health advantages of whole grains through microbiota modification (De Filippo et al., 2010). 

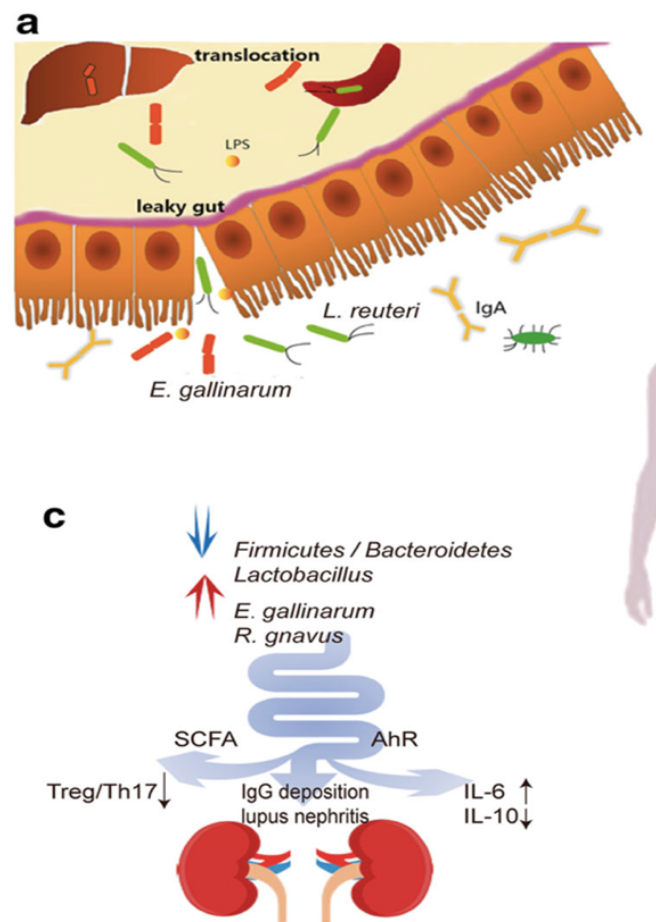

b

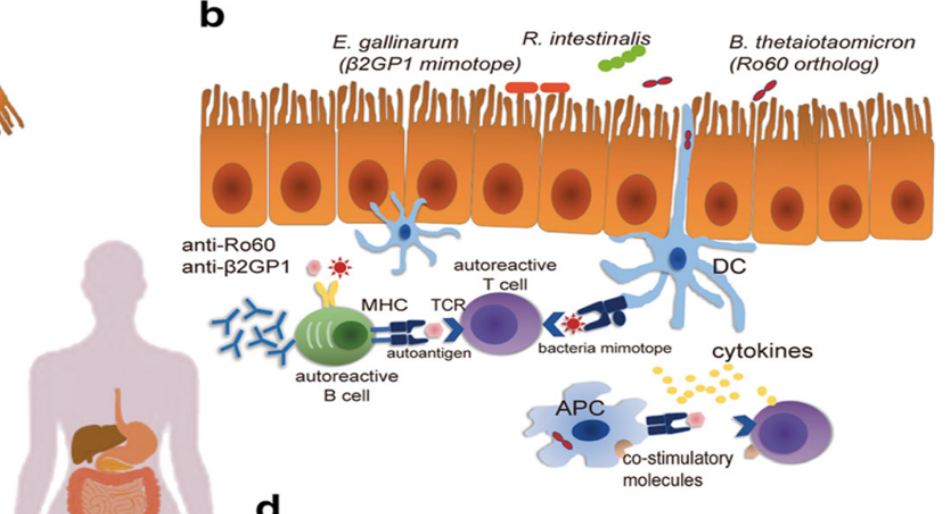

d

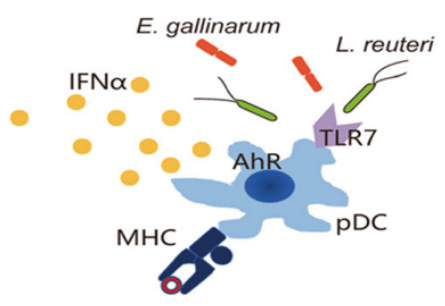

Fig. 1. The pathogenesis of SLE from gut microbial. (a) \& (c) SLE patients have a limited microbial diversity in their stomach, with the possibility of expanding their microbiome. (a) Microbiota can be translocated to MLNs and the liver when gut permeability is compromised. (b) E. gallinarum delivers ligands to the aryl hydrocarbon receptor (AhR), and (d) Proliferation of Th17 and Tfh cells is stimulated by activation of the AhR pathway, which leads to the generation of systemic autoantibodies. E. gallinarum causes plasmacytoid dendritic cells (pDCs) and hepatocytes to produce type I interferon (IFN). TLR7-dependent L. reuteri translocation increases the amount of pDCs and type I IFN expression, inducing SLE. Cross-reactive T and B cell responses are triggered by bacterial orthologs' molecular mimicry of human autoantigens, causing SLE.

Of fact, there is no other study in the literature that specifically evaluates the impact of the amount and type of lipids in the diet on the microbiota of people with SLE. Cuervo et al found no evidence of a link between lipids or saturate fatty acids in moderate levels in a well-balanced diet and fecal microbiota in an SLE cohort in a descriptive investigation (Cuervo et al., 2015). In a previous study conducted recently, researchers found a link between fruit consumption, such as oranges and apples, and reductions in specified microbe populations in SLE patients (Cuervo et al., 2015). It's crucial to remember that these fruits are natural sources of polyphenols and fibers with a significant potential for microbiota modulation. Bifidobacteria degrades particular phenolic chemicals, such as dihydrochalcones from apples, as well as dietary fiber, encouraging its proliferation. Given the immunomodulatory impact attributed to several strains of the genus Bifidobacterium, these types of alterations might be particularly interesting for SLE patients (Konieczna et al., 2012). The Bifidobacterium bifidum LMG13195 strain, for example, increased the proliferation of Treg cells, a type of T-cell that promotes mucosal homeostasis (Lopez et al., 2011). Another study discovered a correlation between dietary flavones and the levels of Blautia sp., a Clostridium cluster XIVA member. Because this microbiota is also involved in Treg cell development, boosting its growth through nutrition may be beneficial in restoring or maintaining immunological activation systems (Atarashi et al., 2011).

Intriguingly, in animal models, a dietary therapy with vitamin A supplementation has demonstrated benefits in SLE pathogenesis, particularly in the decrease of both proteinuria and glomerulonephritis, and has been found to lessen symptoms generally (Hsieh \& Lin, 2011). Another dietary intervention research found that retinoic acid (the active form of vitamin A - retinol) supplementation restored decreased lactobacilli levels that had fallen during the development of SLE in an animal model (Cuervo et al., 2015).

\section{B. Probiotics or Prebiotics}

Surprisingly, taking probiotics or prebiotics has been shown to increase gut microbial health in people with SLE. Another study found that long-term probiotic consumption may lower lupus severity by regulating inflammatory responses and reducing autoantibody synthesis (Esmaeili et al., 2017). When compared to microbiota isolated from healthy controls, microbiota isolated from SLE patient feces samples boosted lymphocyte activation and naïve CD4 + lymphocyte differentiation towards the Th17 subset (Lopez et al., 2011), based on a study by López et al., (2011) Furthermore, when the same proportions of Bifidobacterium bifidum LMG13195 $(\mathrm{Bb})$ or a mix of two Clostridia strains (CI:Ruminococcus obeum DSM 25238 and Blautia coccoides DSM935) substituted 5, 10, or 30\% of SLE gut microbiota, it was found that $\mathrm{Bb}$ and $\mathrm{CI}$ notably reduced $\mathrm{CD} 4+$ lymphocyte over-activation and Th17/Th1 balance (Lopez et al., 2011). Both BB and CI are well-known for their Treg inducing effects (Lopez et al., 2011). Mike et al., (1999) found that feeding mice a Lactobacillus casei (LC) diet from the time they were weaned might extend their lives and inhibit the growth of B220+ T lymphocytes in the spleen and mesenteric lymph nodes (Mike et al., 1999). In addition, intraperitoneal injection of LC into mice dramatically reduced splenocyte proliferation and the B220+CD4-CD8-T cells accumulation, while boosting I-A-macrophages and macrophage colonyforming cells (M-CFCs) (Mike et al., 1999).

Overall, while there is a paucity of data on SLE therapy based on gut microbiota strategy, the present evidence leads to a feasible SLE treatment method (Esmaeili et al., 2017). 
However, the underlying processes causing the beneficial effects of a particular diet, prebiotics, or probiotics on SLE are yet unknown, and further comprehensive study is needed (Pigneur \& Ruemmele, 2019).

\section{CONCLUSION}

According to this review, gut microbiota imbalance can cause gut barrier problems and microbial translocation, which leads to immune dysregulations such as IFN pathway overactivation and Th17/Treg imbalance. These conditions are linked to SLE pathophysiology by inducing self-tolerance breakdown and autoantibody production. Individualized gut microbiota management, such as dietary intervention and probiotic or prebiotic supplementation, might be a potential SLE therapeutic techniques in the future. However, more research is needed to assess the long-term benefit of the dietary strategy on SLE patients.

\section{REFERENCES}

Schulz, S.W., \& Derk, C.T. (2009). The gastrointestinal manifestations of systemic lupus erythematosus: a survey of the literature. The Open Autoimmunity Journal, 1: 10-26.

Azzouz, D., Omarbekova, A., Heguy, A., Schwudke, D., Gisch, N., Rovin, B.H., et al. (2019). Lupus nephritis is linked to disease-activity associated expansions and immunity to a gut commensal. Ann. Rheum. Dis, 78: 947-56.

Atarashi, K., Tanoue, T., Shima, T., Imaoka, A., Kuwahara, T., Momose, Y., et al. (2011). Induction of colonic regulatory $\mathrm{T}$ cells by indigenous Clostridium species. Science, 331: 337-41

Bandre, A., Subramanian, S., Mahajan, S. (2021). Role of gut microbiota in autoimmune diseases: a review. J Vaccines Immunol, 6 (163): 1-14.

Cuervo, A., Hevia, A., Lopez, P., Suarez, A., Sanchez, B., Margolles, A., et al. (2015). Association of polyphenols from oranges and apples with specific intestinal microorganisms in systemic lupus erythematosus patients. Nutrients, 7: 1301-17.

De Filippo, C., Cavalieri, D., Di Paola, M., Ramazzotti, M., Poullet, J.B., Massart, S., et al. (2010). Impact of diet in shaping gut microbiota revealed by a comparative study in children from Europe and rural Africa. Proc Natl Acad Sci, 107: 14691-6.

Esmaeili, S.A., Mahmoudi, M., Momtazi, A.A., Sahebkar, A., Doulabi, H., Rastin, M. (2017). Tolerogenic probiotics: potential immunoregulators in systemic lupus erythematosus. J Cell Physiol, 232(8): 1994-2007.

Gronwall, C., Chen, Y., Vas, J., Khanna, S., Thiel, S., Corr, M., et al. (2012). MAPK phosphatase-1 is required for regulatory natural autoantibodymediated inhibition of TLR responses. Proc Natl Acad Sci USA, 109: 19745-50.

Han, S., Zhuang, H., Shumyak, S., Yang, L., Reeves, W.H. (2015). Mechanisms of autoantibody production in systemic lupus erythematosus. FrontImmunol, 6: 228.

Hevia, A., Lopez, P., Suarez, A., Jacquot , C, Urdaci, M.C, Margolles, A., et al. (2014). Association of levels of antibodies from patients with inflammatory bowel disease with extracellular proteins of food and probiotic bacteria. Biomed Res Int, 1-8.

Hollister, E.B., Gao, C., Versalovic, J. (2014). Compositional and functional features of the gastrointestinal microbiome and their effects on human health. Gastroenterology, 146: 1449-58.

Hevia, A., Milani, C., Lopez, P., Cuervo, A., Arboleya, S., Duranti S., et al. (2014). Intestinal dysbiosis associated with systemic lupus erythematosus. Microbiology, 5: e01548-14.

Hsieh, C.C., \& Lin, B.F. (2011). Dietary factors regulate cytokines in murine models of systemic lupus erythematosus. Autoimmun Rev, 11: 22-7.

Kamada, N., \& Núnez, G. (2014). Regulation of the immune system by the resident intestinal bacteria. Gastroenterology, 146: 1477-88.

Konieczna, P., Akdis, C.A., Quigley, E.M., Shanahan, F., O’Mahony, L. (2012). Portrait of an immunoregulatory Bifidobacterium. Gut Microbes, 3: 261-6.

Lopez, P., de Paz, B., Rodriguez-Carrio, J., Hevia, A., Sanchez, B., Margolles, A., et al. (2016). Th17 responses and natural IgM antibodies are related to gut microbiota composition in systemic lupus erythematosus patients. Sci Rep, 6: 24072.
Lopez, P., Gonzalez-Rodriguez, I., Gueimonde, M., Margolles, A., Suarez, A. (2011). Immune response to Bifidobacterium bifidum strains support Treg/Th17 plasticity. PLoS One, 6: e24776.

Ley, R.E., Turnbaugh, P.J., Klein, S., Gordon, J.I. (2006). Microbial ecology - human gut microbes associated with obesity. Nature, 444: 1022-3.

Mu, Q., Zhang, H., Liao, X., Lin, K., Liu, H., Edwards, M.R., et al. (2017). Control of lupus nephritis by changes of gut microbiota. Microbiome, 5(73): 1-12

Mahajan, P., Kulkarni, A., Bandre, A., Subramanian, S., Mahajan, S. (2021). Role of gut microbiota in autoimmune diseases: a review. $J$ Vaccines Immunol, 6(163): 1-14.

Mu, Q., Zhang, H., Luo, X.M. (2015). SLE: Another autoimmune disorder influenced by microbes and diet?. Front Immunol, 6: 608 .

Ma, Y., Xu, X., Li, M., Cai, J., Wei, Q., Niu, H. (2019). Gut microbiota promote the inflammatory response in the pathogenesis of systemic lupus erythematosus. Molecular Medicine, 25(35): 1-16.

Manfredo, V.S., Hiltensperger, M., Kumar, V., Zegarra-Ruiz, D., Dehner, C., Khan, N., et al. (2018). Translocation of a gut pathobiont drives autoimmunity in mice and humans. Science, 359(6380): 1156-61.

Mu, Q., Tavella, V.J., Kirby, J.L., Cecere, T.E., Chung, M., Lee, J., et al. (2017). Antibiotics ameliorate lupus-like symptoms in mice. Sci Rep, 7(1): 13675

Martínez, I., Lattimer, J.M., Hubach, K.L., Case, J.A., Yang, J., Weber, C.G., et al. (2013). Gut microbiome composition is linked to whole graininduced immunological improvements. ISMEJ, 7: 269-80.

Mike, A., Nagaoka, N., Tagami, Y., Miyashita, M., Shimada, S., Uchida, K., et al. (1999). Prevention of B220+ T cell expansion and prolongation of lifespan inducedby Lactobacillus Casei in MRL/Lpr mice. Clin Exp Immunol, 117(2): 368-75.

Neuman, H., \& Koren O. (2017). The gut microbiota: a possible factor influencing systemic lupus erythematosus. Curr Opin Rheumatol, 29: 374-77.

Ogunrinde, E., Zhou, Z., Luo, Z., Alekseyenko, A., Li, Q.Z., Macedo, D., et al. (2019). A link between plasma microbial translocation, microbiome, and autoantibody development in first-degree relatives of systemic lupus erythematosus patients. Arthritis Rheumatol, 71(11): 1858-68.

Perry, M., Swain, S., Kemmis-Betty, S., Cooper, P. (2014). Guideline development group. multiple sclerosis: summary of NICE guidance. $B M J, 349: 5701$.

Pigneur, B., \& Ruemmele, F.M. (2019). Nutritional interventions for the treatment of IBD: current evidence and controversies. Therap Adv Gastroenterol. 2019; 12: 1756284819890534.

Rees, F., Doherty, M., Grainge, M.J., Lanyon, P., Zhang, W. (2017). The worldwide incidence and prevalence of systemic lupus erythematosus: a systematic review of epidemiological studies. Rheumatology (Oxford), 56(11), 1945-61.

Schulz, S.W., \& Derk, C.T. (2009). The gastrointestinal manifestations of systemic lupus erythematosus: a survey of the literature. The Open Autoimmunity Journal, 1:10-26.

Thim-Uam, A., Surawut, S., Issara-Amphorn, J., Jaroonwitchawan, T., Hiengrach, P., Chatthanathon, P., et al. (2020). Leaky-gut enhanced lupus progression in the Fc gamma receptor-IIb deficient and pristane induced mouse models of lupus. Sci Rep, 10(1): 777.

Vieira, S.M., Pagovich, O.E., Kriegel, M.A. (2014). Diet, microbiota and autoimmune diseases. Lupus, 23: 518-26.

Wei, F., Xu, H., Yan, C., Rong, C., Liu, B., Zhou, H. (2019). Changes of intestinal flora in patients with systemic lupus erythematosus in northeast China. PLOS ONE, 14(3): 1-11.

Wu, G.D., Chen, J., Hoffmann, C., Bittinger, K., Chen, Y.Y., Keilbaugh, S.A., et al. (2011). Linking long-term dietary patterns with gut microbial enterotypes. Science, 334: 105-8.

Yatsunenko, T., Rey, F.E., Manary, M.J., Trehan, I., Dominguez-Bello, M.G., Contreras, M., et al. (2012). Human gut microbiome viewed across age and geography. Nature, 486: 222-7.

Zegarra-Ruiz, D.F., El Beidaq, A., Iniguez, A.J., Lubrano, R. M., Manfredo, V. S., Ruff, WE., et al. (2019). A diet-sensitive commensal Lactobacillus strain mediates TLR7-dependent systemic autoimmunity. Cell Host Microbe, 25(1): 113-27. 